\title{
Reducing cardiovascular disease in a rural community
}

\author{
Sallie D. Allgood PhD, MS, RN ${ }^{1}$ (D) | Jennifer Leeman DrPH, MDiv ${ }^{1,2}$ | Cherie A. Rosemond \\ $\mathrm{PhD}^{3,4}$ | Alice S. Ammerman DrPH ${ }^{2,4}$ | Carmen Samuel-Hodge PhD, MS, RD ${ }^{2,4}$ | \\ Samuel Cykert MD ${ }^{2,5}$
}

\author{
${ }^{1}$ School of Nursing, The University of North \\ Carolina at Chapel Hill, Chapel Hill, North \\ Carolina \\ ${ }^{2}$ Center for Health Promotion and Disease \\ Prevention, The University of North Carolina \\ at Chapel Hill, Chapel Hill, North Carolina \\ ${ }^{3}$ Partnerships in Aging Program, The \\ University of North Carolina at Chapel Hill, \\ Chapel Hill, North Carolina \\ ${ }^{4}$ Department of Health Behavior, Gillings \\ School of Global Public Health, The \\ University of North Carolina at Chapel Hill, \\ Chapel Hill, North Carolina \\ ${ }^{5}$ Division of General Medicine and Clinical \\ Epidemiology, School of Medicine, The \\ University of North Carolina at Chapel Hill, \\ Chapel Hill, North Carolina \\ Correspondence \\ Sallie D. Allgood, The University of North \\ Carolina at Chapel Hill, School of Nursing, \\ Carrington Hall, Campus Box \#7460, Chapel \\ Hill, NC 27599-7460. \\ Emails: sallie.allgood@duke.edu; \\ allgood@gmail.com \\ Funding information \\ Centers for Disease Control and Prevention, \\ Grant/Award Number: U48/DP005017; \\ National Institute of Nursing Research, \\ Grant/Award Number: T32NR007091
}

\begin{abstract}
In this case report, we describe the one-year formative phase of a five-year study to develop, implement, and test a community health worker (CHW)-delivered cardiovascular disease (CVD) prevention intervention. The purpose of the formative phase was to engage community partners in the adaptation of an existing evidence-based CVD prevention intervention to fit the needs and preferences of a rural, predominantly African-American community. The formative work was guided by a framework for adapting evidence-based interventions and involved engaging stakeholders in assessing the intervention's fit with the local context and then applying assessment findings to iteratively adapt the intervention's contents, materials, and delivery methods. Findings from the formative work were then applied to develop $\mathrm{CHW}$ position descriptions, workflow diagrams, and a training plan. Findings also were applied to adapt intervention materials and protocols to fit the needs of the community. This case report illustrates how community-engaged formative work can be applied to adapt an evidence-based intervention to fit community needs and resources.
\end{abstract}

\section{KEYWORDS}

African-Americans, cardiovascular diseases, case report, community health workers, evidence-based intervention, health behavior, health promotion, rural health

\section{1 | BACKGROUND}

Cardiovascular disease (CVD) affects more than one in three Americans, and is the leading cause of death in the United States (Benjamin et al., 2017). Rural African-Americans have higher rates of CVD and worse outcomes when compared to the general population (Benjamin et al., 2017; O'Connor \& Wellenius, 2012; Williams, Priest, $\&$ Anderson, 2016). Much of the risk for developing CVD is attributable to modifiable factors that can be reduced through interventions that promote risk screening, medication use, and health behavior change (Benjamin et al., 2017; Patnode, Evans, Senger, Redmond, \&
Lin, 2017). However, these interventions are not reaching the most at-risk populations, particularly those in rural communities where access to health care is less reliable, health care quality is lower, and community resources are more scarce than in urban communities (Singh \& Siahpush, 2014a, 2014b). To reduce CVD burden in rural communities, interventions need to be culturally relevant, tailored to community needs and assets, and implemented through engaged partnership with members of the community (Cooper, Hill, \& Powe, 2002). In this paper, we describe the year of formative work done to engage community and academic partners in the development of a community health worker ( $\mathrm{CHW}$ )-delivered intervention to modify 
CVD risk factors in a predominantly African-American, rural community. The intervention was designed to employ $\mathrm{CHWs}$ in a local Federally Qualified Health Center (FQHC) and Department of Public Health (DPH).

FQHCs care for underserved populations across the United States with the goal of reducing health disparities (Nath, Costigan, \& Hsia, 2016). FQHCs are well equipped to screen for CVD risk factors and to prescribe medications (Hung et al., 2007; Institute of Medicine, 2012); however, they often lack the capacity to provide health behavior change counseling (Etz et al., 2008; US Preventive Services Task Force, 2014). In response to lack of capacity, the Institute of Medicine (2012) and others recommend providers refer patients to health departments, cooperative extension services, and other community resources to gain additional support for behavior change (CDC, 2017; Ferdinand et al., 2012; US Preventive Services Task Force, 2014). Yet, providers face multiple challenges in their efforts to link patients to community resources including limited time, competing priorities, and limited information on available community resources (Etz et al., 2008; Hung et al., 2007; Lebrun et al., 2012; Porterfield et al., 2012). In contrast to FQHCs, many $\mathrm{DPH}$ have resources to support behavior change and have the further advantage of reaching underserved populations. The Carolina Heart Alliance Networking for Greater Equity (CHANGE) intervention sought to integrate the strengths of FQHCs and DPH, using a community-engaged approach to intervention development.

A growing body of research provides guidance on how to build community-clinical linkages (Porterfield et al., 2012). This guidance suggests that linkages are most effective when "a cultivation period" allows partners to establish a trusting, collaborative relationship and to define their respective responsibilities and contributions (Lebrun et al., 2012). Success is also enhanced when partners perceive the effort to be feasible and acceptable (Etz et al., 2008). An additional strategy to forge community-clinical linkages is the engagement of $\mathrm{CHWs}$ to connect community members with health care and to connect clinics with community resources (Quigley, Matsuoka, Montgomery, Khanna, \& Nolan, 2014).

The American Public Health Association (2009) defines a CHW as a "frontline public health worker who is a trusted member of and/ or has an unusually close understanding of the community served." The Guide to Community Preventive Services Task Force (2015) recommends engaging CHWs in CVD prevention efforts and has identified a need for more research on $\mathrm{CHWs}$ in rural communities. Although $\mathrm{CHW}$-led interventions have demonstrated effectiveness at improving outcomes across a range of diseases, these studies provide limited information on $\mathrm{CHWs}$ training and the roles $\mathrm{CHWs}$ play in forging community-clinical linkages (Lohr, Ingram, Nuñez, Reinschmidt, \& Carvajal, 2018).

In this paper, we describe the year of formative work done to engage community and academic partners in the development of CHANGE, a CHW-led CVD-prevention intervention. The study's community-engaged planning team completed this work during the first year of a five-year study to test the CHANGE intervention in one rural community (Years 2-3) and replicate it in a second rural community (Years 4-5). During the formative year, engaged partners adapted an existing evidence-based CVD-prevention intervention (Heart-to-Health) to be culturally appropriate to the local community and to be delivered by $\mathrm{CHWs}$. The intervention involves in-person counseling sessions and phone calls that walk participants through a handbook of educational materials and prompts to set goals for quitting tobacco use and improving dietary intake, physical activity, and medication adherence (Keyserling et al., 2014).

\section{1 | Conceptual framework for adaptation}

Formative work was guided by steps that Escoffery et al. (2019) identified for adapting an intervention to fit a new population and context. Those steps include (1) assessing the intervention's fit with the local population's culture, organizational systems, and community resources; (2) adapting the intervention's content, materials, and delivery; (3) piloting the adaptations with community members; (4) and developing a staff training plan. The assessment of fit (Step 1) was further guided by Andersen's Behavioral Model of Health Services Use, which describes how environmental factors (e.g. cultural norms, community resources) and characteristics of a population (e.g. education levels, income) pre-dispose and enable healthy behaviors and use of health services (Andersen, 1995).

\section{2 | Purpose}

In this paper, we report on Phase 1, the "cultivation period," of the CHANGE intervention (10/2014-10/2015), which addressed the following aims:

1. adapt an evidence-based intervention (Heart-to-Health) to the needs and preferences of the local community;

2. create a comprehensive inventory of community resources to support healthy eating, physical activity, smoking cessation, and medication adherence;

3. develop the $\mathrm{CHW}$ role and position description;

4. diagram the workflow for identifying and referring community members to $\mathrm{CHWs}$ and linking them to community resources; and

5. design a $\mathrm{CHW}$ training plan.

\section{2 | METHODS}

\section{1 | Setting}

The formative work was done in a rural North Carolina county that is $59.2 \%$ African-American and $36.4 \%$ Caucasian and has an age adjusted CVD death rate of 259.2 per 100,000 residents as compared to 221.9 for North Carolina overall. The county has high rates of poverty (over $30 \%$ of residents) and is ranked $93 \mathrm{rd}$ of 100 North Carolina counties for health behaviors; among the county's adult population, $27 \%$ are smokers, $33 \%$ are obese, and $33 \%$ are physically 
inactive (County Health Rankings, 2015; North Carolina State Center for Health Statistics, 2017).

\subsection{Data collection}

The formative phase included meetings of a community-engaged planning team, in-depth interviews with key stakeholders, geographical information systems (GIS) mapping, and interviews with community members to assess the acceptability of intervention materials and delivery methods. The University's Institutional Review Board approved the study.

\subsection{1 | Meetings of the community-engaged planning team}

A community-engaged planning team was created and met monthly throughout the project's formative phase. The academic researchers identified team members by reaching out to leaders of the county's FQHC and DPH and then working with these leaders to identify other organizations in the county that provide health care or behavior change services for low-income community members. The final team included representatives from the FQHC, DPH, Cooperative Extension, community college, hospital, and wellness center as well as representatives from the University of North Carolina Schools of Nursing, Medicine, and Public Health. The CHW supervisors were also members of the planning team and included a nurse at the FQHC and a health educator from the $\mathrm{DPH}$. CHWs were not hired until the second year of the study and therefore did not attend the planning meetings. A faculty member with expertise in community-engaged research facilitated the monthly team meetings to plan, design, and adapt the intervention and to review and revise iterative versions of intervention content, materials, approach to delivery and other documents (e.g., $\mathrm{CHW}$ training plan, work flow diagrams, and position description).

\subsection{2 | In-depth interviews with key stakeholders}

The community-engaged planning team identified 10 key informants with extensive experience providing clinical and behavior-change related services to members of the community. Participants included employees of the FQHC, the hospital, a non-profit health network, a local wellness center, the County Office of Aging, the DPH, and the Cooperative Extension. With input from the planning team, a researcher with expertise in qualitative methods $(\mathrm{JL})$ developed a structured interview guide that was designed to explore the interrelated factors detailed in Andersen's framework, with the goal of understanding how they contributed to CVD risk (Andersen, 1995). Questions addressed relationships across organizations, how referrals to health resources were made, what community resources were available, and what barriers impeded community members' use of available health and community resources. Interviews were audio recorded and lasted between 30 and $45 \mathrm{~min}$.

\subsection{3 | GIS mapping}

ArcGIS (Version 10; Esri) was used to create an initial map of the county with the locations of parks, recreational facilities, and food retailers. The map was presented to the community-engaged planning team and input solicited on where the geographic boundaries of the community should be drawn. The team was also tasked with identifying additional resources that should be included to support participants' efforts to improve their dietary, physical activity, tobacco use, and medication adherence behaviors. Team members also conducted windshield tours to identify locations of community resources. A research associate then interviewed staff at each of the identified resources to collect information on hours of operation, services offered, cost of services, eligibility requirements, and contact information that $\mathrm{CHWs}$ or participants could use to learn more. Additionally, staff at these community resources were asked to identify other resources in the community.

\subsection{4 | Acceptability interviews with community members}

Input was sought on the intervention materials and delivery method from nine community members representative of the population intended to benefit from the intervention. A research associate gathered input through visits to the $\mathrm{FQHC}$ and wellness center where she invited older (50 years and up) and primarily African-American community members to be interviewed and offered a $\$ 20$ incentive to compensate them for their time. She conducted interviews using a structured interview guide to elicit feedback. Questions addressed community members' response to $\mathrm{CHWs}$ coming into their homes and calling them and their perceptions of the intervention materials. Interviews were audio recorded and lasted between 20 and 30 min.

\section{3 | Analytic strategy}

Minutes were taken at the community-engaged planning team meetings. Between meetings, investigators reviewed minutes and revised protocols and documents to incorporate team input. Audio recordings of key stakeholder interviews were transcribed and four investigators reviewed transcripts and identified themes independently. The four investigators then met to review themes and achieve consensus on local barriers and facilitators to clinical care, healthy behaviors, and community-clinical linkages (Miles \& Huberman, 1994). The themes were presented to the communityengaged planning team for their reflection and input. GIS and interview data on community resources were compiled into a summary document. To analyze the acceptability interviews, two investigators independently reviewed audio-recordings of interviews and summarized community members' observations and suggested revisions to the intervention approach and materials. They then met to review and reconcile their findings, which were then summarized and presented to the community-engaged planning team. The findings were then applied to guide adaptations to the intervention materials and protocols. 
TAB LE 1 How formative findings were applied to adapt intervention components

\begin{tabular}{|c|c|c|}
\hline Intervention Components & Formative findings on fit & How adapted to fit \\
\hline $\mathrm{CHW}$ role and position description & $\begin{array}{l}\text { - DPH experienced with } \mathrm{CHWs} \text { reaching out } \\
\text { to underserved populations } \\
\text { - Community distrust of outsiders } \\
\text { - Limited access to transportation } \\
\text { - CHWs visits no longer than } 90 \mathrm{~min}\end{array}$ & $\begin{array}{l}\text { - DPH CHWs focus on outreach to } \\
\text { underserved } \\
\text { - DPH CHWs partner with community set- } \\
\text { tings (e.g., churches) } \\
\text { - } \mathrm{CHWs} \text { hired from community } \\
\text { - } \mathrm{CHWs} \text { make < 90-min home visits }\end{array}$ \\
\hline $\begin{array}{l}\text { Workflow for participant identification and } \\
\text { referral }\end{array}$ & - Only FQHC-based CHWs had access to EHR & $\begin{array}{l}\text { - Distinct workflows for FQHC and DPH } \\
\text { CHWs }\end{array}$ \\
\hline CHW training & $\begin{array}{l}\text { - Content to be covered } \\
\text { - Local experts in identified content areas }\end{array}$ & $\begin{array}{l}\text { - Recruited local experts to co-deliver the } \\
\text { training }\end{array}$ \\
\hline Heart-to-Health handbook and protocols & $\begin{array}{l}\text { - Low literacy levels } \\
\text { - Limited resources } \\
\text { - Photos need to represent community } \\
\text { - Community members did not eat cashews } \\
\text { and had local food traditions that featured } \\
\text { herring and boiled peanuts }\end{array}$ & $\begin{array}{l}\text { - Reduce and simplify educational text } \\
\text { - Highlight low-cost options for dietary and } \\
\text { physical activity } \\
\text { - Added photos of African-American men of } \\
\text { all ages } \\
\text { - In some cases, replaced existing photos with } \\
\text { local foods and products }\end{array}$ \\
\hline Inventory of community resources & $\begin{array}{l}\text { - Strong intra-organizational relationships } \\
\text { - Community resources to support CVD } \\
\text { prevention }\end{array}$ & $\begin{array}{l}\text { - Inventory included resources and their } \\
\text { locations, services offered, hours, costs, and } \\
\text { contact information }\end{array}$ \\
\hline
\end{tabular}

\section{3 | RESULTS}

Below we present the findings that resulted from each method used and how they were applied to develop and/or adapt the intervention components (See Table 1 for an overview).

\section{1 | Findings from meetings with the engaged planning team}

The community-engaged planning team developed the $\mathrm{CHW}$ role and position descriptions, diagrams of workflows for participant identification and referral, and the $\mathrm{CHW}$ training plan. Through these meetings we learned that the FQHC had an electronic health record (EHR) based referral system that could be adapted for referral of patients to CHWs and that the CHW at the DPH would not have access to the FQHC's EHR. We also learned that the DPH had a strong history of working with $\mathrm{CHWs}$ to reach underserved populations through community outreach. Based on these findings, the workflow diagram for the FQHC-based $\mathrm{CHW}$ involved a staff person flagging at-risk patients and then health care providers referring those patients to the $\mathrm{CHW}$. The workflow diagram for the DPH-based $\mathrm{CHW}$ involved the $\mathrm{CHW}$ going into the community to identify and enroll at-risk community members in the CHANGE intervention and then referring community members to the FQHC if they did not already have a primary care provider. The two differing workflows made it difficult for $\mathrm{CHWs}$ to cover for each other during absences and the team therefore made the decision to have two part-time $\mathrm{CHWs}$ based at each location in future replications of the intervention.

The community-engaged planning team also created a six-day CHW training plan. The first 4 days provided general information about the cardiovascular system, CVD prevention, and healthy behaviors (i.e., physical activity, nutrition, smoking cessation, and medication adherence). This initial training also included information about working with clients and topics like cultural competency, adult learning and behavior change theories, lifestyle counseling, home visiting safety, generational poverty, and an overview of community resources. Following the initial 4-day training, $\mathrm{CHWs}$ were given time to familiarize themselves with the intervention content and community resources. The additional two days of training then included content on research ethics and study protocols and also provided an opportunity for $\mathrm{CHWs}$ to practice delivering the intervention. To develop the training, the community-engaged planning team identified community stakeholders with expertise in key content areas who then co-delivered the training with members of the planning team. Both $\mathrm{CHWs}$ and their supervisors attended the highly interactive training.

\subsection{Findings from interviews with key stakeholders}

The following themes emerged from stakeholder interviews: (a) community organizations have strong relationships; (b) community members were perceived to have low literacy levels, limited resources, and a distrust of outsiders; and (c) the community transportation environment limits access to community and clinical services.

\subsubsection{Community organizations have strong relationships}

Most of the organizations participating in the study had a history of prior collaborations and worked in a coalition to increase community 
members' access to health care services and resources. In the words of one stakeholder,

I keep emphasizing this, but our area is really small so we all depend on each other. You see the same people on the committees. We partnered with them on our community assessment. They are a referral base for us and we are for them ... I know these programs are needed in our community, so we work very hard together. Our agencies are short staffed. We don't hear no's and we know how to work together.

The history of collaboration between community organizations to improve the health of the community was an asset that supported the development of shared goals around the adaptation and implementation plan for the CHANGE intervention.

\subsubsection{Community members were perceived to have low literacy levels, limited resources, and a distrust of outsiders}

Stakeholders reported that many community members have low literacy levels. Because of the limited employment options in the county, many young people leave after graduating high school and many who stay lack a high school diploma. One stakeholder noted, "If you stick 25 of my clients together, I bet 15 have not graduated from high school... They don't have the ability to read...." Stakeholders also identified a lack of financial and other resources as a barrier to healthy behavior. As one stakeholder reported, "A lot of people are not able to buy the type of food and engage in the type of behaviors that promote a healthy lifestyle." Many people got food from food pantries, which tend to carry products with a long shelf life as opposed to fresh or frozen fruits and vegetables. Community members also were suspicious of help that comes from sources outside the county. In the words of one interviewee, "... there is a tremendous amount of distrust. People wonder, what is the catch? Especially if it is free." They advised that, to work with this community, members of the CHANGE intervention team needed to connect with individuals who will sponsor them and invite them into their "communities within communities" to "get someone in that community who will tell someone else that you are safe." Churches, bike shows, tattoo parlors, car shows, barbershops, and beauty shops were all identified as communities within the larger community. We also learned that the community valued the role of $\mathrm{CHWs}$ based on their experience with prior $\mathrm{CHW}$ initiatives such as HIV peer counseling and breastfeeding support programs.

\subsection{3 | The community's transportation environment limits access to community and clinical services}

All stakeholders mentioned the challenges that result from living in a rural county where long distances separate organizations, services, and community members. Distance affected access to care, access to food, and access to employment. Many community members lacked personal transportation or had limited funds for gas. Public transportation was limited to a mini-bus service for medical appointments that required a three- to five-day advance notice for scheduling.

Based on findings from stakeholder interviews, the research team revised the Heart-to-Health educational and goal setting materials to reduce and simplify the written text and to highlight low cost options for dietary intake and physical activity. They adapted the $\mathrm{CHW}$ training plan to reinforce the importance of verbally delivering the materials and engaging participants in discussing how they might apply the information in their day-to-day lives. Lastly, the findings confirmed the team's decision to have CHWs travel to participants' homes, unless the participant requested another location.

\section{3 | GIS mapping}

GIS mapping, together with windshield tours and input from the planning team, yielded a list of 74 community resources that supported CVD prevention and other community needs. General health resources were identified and included disease specific classes, support groups, and employee wellness programs. Clinically related resources were also featured such as health care services, insurance, and medication assistance and also included resources for financial help and automated reminder services. To support healthy dietary intake, the list included food stores, markets, meal services, and pantries. The list also included places in the community to be physically active and locations of smoking cessation programs. This list was used to create a community resource guide that provided an overview of each resource, including specific services offered, hours of operation, contact information, and costs.

\subsection{Findings from acceptability interviews with community members}

Community members reported that the intervention's educational and goal-setting materials were clear. They stated they were comfortable having a $\mathrm{CHW}$ visit their homes and thought $\mathrm{CHW}$ visits should be no longer than 90 min. Community members provided specific feedback on ways the materials might be further refined to fit with their experience. In particular, they recommended replacing existing photographs with photographs that better reflected the community's demographics. They also identified specific food and beverage products that were recommended in the manual but were not consumed by the community, with one focus group member stating, "they didn't know anyone who ate those kind of crackers." Based on community members' feedback, the intervention materials were further updated and brought to the community-engaged planning team further discussion. Examples of changes made based on community members' feedback included replacing the recommendations to eat cashews with peanuts, adding herring to the list of fish, and changing food brands in photographs to those available and preferred locally. Additionally, lists of food options were reordered, 
with less expensive options listed first. Also, new photos were added to be more representative of community members, such as photos of older people walking in a park, and of younger black men walking and lifting weights.

\section{4 | DISCUSSION}

In this paper, we report on the formative work to develop community-clinical linkages and engage community members to adapt an existing evidence-based intervention to be delivered by $\mathrm{CHWs}$ to a rural, predominantly African-American community. The formative work was led by a community-engaged planning team with the goal of gaining community input while also further strengthening the clinical and community relationships that are key to forging clinicalcommunity linkages (Etz et al., 2008).

The CHANGE intervention was designed with the goal of spreading the intervention to other rural counties. After the intensive period of intervention development described here, the intervention was implemented (Years 2-3 of the study) and then replicated in a second county (Years 4-5) with the goal of developing the guidance needed for scaling up and spreading the CHANGE intervention to additional rural communities. The goal of many of the adaptations reported here is that these adaptations are generalizable to other rural FQHCs and $\mathrm{DPH}$. The $\mathrm{CHW}$ position descriptions, workflow diagrams, and training plan can be generalized, with some adaptation to fit the needs of new settings. The Heart-to-Health handbook also can be generalized; however, new communities are advised to solicit community member input on the handbook's photos and guidance to maximize fit with local foods and preferences for physical activity. Although the inventory of community resources is not generalizable, the approach used to create it can be replicated in new communities.

The Community Guide recommends that $\mathrm{CHWs}$ be engaged in CVD prevention interventions but has identified a lack of research on CHWs working in rural areas or on CHWs' ability to "build and enhance community-clinical linkages" (Guide to Community Preventive Services Task Force, 2015). This paper reports the formative work done as part of a research study that will add to the small but growing body of research that engages CHWs in rural CVD prevention interventions. This formative work focused on developing the CHANGE intervention so that it built on the existing evidence base for how to most effectively intervene in rural communities and engage $\mathrm{CHWs}$. Prior studies have shown that community partners are valuable when adapting and designing interventions for rural communities (Cornell et al., 2009; Kuhajda et al., 2006), that clinic-community partnerships can be successfully forged (Krantz et al., 2013), that CHWs can deliver CVD-prevention interventions (Cornell et al., 2009; Dye, Williams, \& Evatt, 2015; Krantz et al., 2013; Kuhajda et al., 2006), and that CHW-delivered CVD-prevention interventions can reduce CVD disease risks (Dye et al., 2015). This study adds to this literature by providing an in-depth description of the formative work done to engage community partners in adapting a
$\mathrm{CHW}$-delivered intervention and strengthening clinical-community linkages in a rural community.

\section{1 | Implications for public health nursing}

The CHW workforce is expanding under new employment opportunities made possible nationally by the Patient Protection and Affordable Care Act and through the creation of accountable care organizations and patient-centered medical neighborhoods. Additionally, within North Carolina, there are statewide efforts to recognize and promote the use of CHWs, bringing additional attention to this workforce across the state (Nelson, Money, \& Petersen, 2016). In response to the increased engagement of CHWs in community health care, The Tri-Council for Nursing (2017) released a position statement describing the unique roles of registered nurses (RNs) and CHWs in teambased care and the role of RNs in coordinating the care provided by CHWs. The CHANGE intervention was designed in anticipation of the expanding $\mathrm{CHW}$ workforce and engagement of $\mathrm{CHWs}$ in team-based care settings. Public health nurses have a central role to play in the development the $\mathrm{CHW}$ workforce, particularly when that workforce is deployed to strengthen community-clinical linkages, as is the case in the CHANGE intervention.

\section{5 | CONCLUSION}

The formative work of the CHANGE intervention provided insight into the distinct context, needs, and resources of a rural, predominately African-American community. These findings provide an example of how formative research can build and strengthen clinical and community partnerships and how these partnerships can be used to adapt an intervention to community needs. Future findings from the CHANGE intervention will assess (a) the effectiveness of the adapted intervention at reducing community members' CVD risk and (b) the implementation outcomes of the CHANGE intervention (e.g., fidelity, acceptability, and feasibility).

\section{ORCID}

Sallie D. Allgood (iD https://orcid.org/0000-0002-0329-4572

\section{REFERENCES}

American Public Health Association. (2009). Support for community health workers to increase health access and to reduce health inequities (APHA Policy No. 20091). Washington, DC: Author. Retrieved July 15, 2016, from https://www.apha.org/policiesand-advocacy/public-health-policy-statements/policy-datab ase/2014/07/09/14/19/support-for-community-health-workers-toincrease-health-access-and-to-reduce-health-inequities

Andersen, R. M. (1995). Revisiting the behavioral model and access to medical care: Does it matter? Journal of Health and Social Behavior, 36, 1-10. https://doi.org/10.2307/2137284

Benjamin, E. J., Blaha, M. J., Chiuve, S. E., Cushman, M., Das, S. R., Deo, R., ... Muntner, P. (2017). Heart disease and stroke statistics-2017 
update: A report from the American Heart Association. Circulation, 135, e146-e603. https://doi.org/10.1161/CIR.0000000000000485

Centers for Disease Control and Prevention. (2017). Chronic disease prevention and health promotion: Heart disease and stroke. Retrieved January 21, 2018, from https://www.cdc.gov/chronicdisease/resou rces/publications/aag/heart-disease-stroke.htm

Cooper, L. A., Hill, M. N., \& Powe, N. R. (2002). Designing and evaluating interventions to eliminate racial and ethnic disparities in health care. Journal of General Internal Medicine, 17, 477-486. https://doi. org/10.1046/j.1525-1497.2002.10633.x

Cornell, C. E., Littleton, M. A., Greene, P. G., Pulley, L., Brownstein, J. N., Sanderson, B. K., ... Raczynski, J. M. (2009). A community health advisor program to reduce cardiovascular risk among rural AfricanAmerican women. Health Education Research, 24, 622-633. https:// doi.org/10.1093/her/cyn063

County health rankings. (2015). Retrieved July 21, 2017, from http:// www.countyhealthrankings.org/app/north-carolina/2015/ranki ngs/hertford/county/outcomes/

Dye, C. J., Williams, J. E., \& Evatt, J. H. (2015). Improving hypertension self-management with community health coaches. Health Promotion Practice, 16, 271-281. https://doi.org/10.1177/1524839914533797

Escoffery, C., Lebow-Skelley, E., Udelson, H., Boing, E. A., Wood, R., Fernandez, M. E., \& Mullen, P. D. (2019). A scoping study of frameworks for adapting public health evidence-based interventions. Translational Behavioral Medicine, 9, 1-10. https://doi.org/10.1093/ tbm/ibx067

Etz, R. S., Cohen, D. J., Woolf, S. H., Holtrop, J. S., Donahue, K. E., Isaacson, N. F., ... Olson, A. L. (2008). Bridging primary care practices and communities to promote healthy behaviors. American Journal of Preventive Medicine, 35, S390-S397. https://doi.org/10.1016/j.amepre.2008.08.008

Ferdinand, K. C., Patterson, K. P., Taylor, C., Fergus, I. V., Nasser, S. A., \& Ferdinand, D. P. (2012). Community-based approaches to prevention and management of hypertension and cardiovascular disease. The Journal of Clinical Hypertension, 14, 336-343. https://doi. org/10.1111/j.1751-7176.2012.00622.x

Guide to Community Preventive Services Task Force. (2015). Cardiovascular disease: Interventions engaging community health workers. Retrieved July 28, 2017, from http://www.thecommunitygui de.org/cvd/RRCHW.html

Hung, D. Y., Rundall, T. G., Tallia, A. F., Cohen, D. J., Halpin, H. A., \& Crabtree, B. F. (2007). Rethinking prevention in primary care: Applying the chronic care model to address health risk behaviors. The Milbank Quarterly, 85, 69-91. https://doi.org/10.1111/j.1468-0009.2007.00477.x

Institute of Medicine. (2012). Primary care and public health: Exploring integration to improve population health. Washington, DC: National Academies Press.

Keyserling, T. C., Sheridan, S. L., Draeger, L. B., Finkelstein, E. A., Gizlice, Z., Kruger, E., ... Ammerman, A. S. (2014). A comparison of live counseling with a web-based lifestyle and medication intervention to reduce coronary heart disease risk: A randomized clinical trial. JAMA Internal Medicine, 174, 1144-1157. https://doi.org/10.1001/jamai nternmed.2014.1984

Krantz, M. J., Coronel, S. M., Whitley, E. M., Dale, R., Yost, J., \& Estacio, R. O. (2013). Effectiveness of a community health worker cardiovascular risk reduction program in public health and health care settings. American Journal of Public Health, 103(1), e19-e27. https://doi. org/10.2105/AJPH.2012.301068

Kuhajda, M. C., Cornell, C. E., Brownstein, J. N., Littleton, M. A., Stalker, V. G., Bittner, V. A., ... Raczynski, J. M. (2006). Training community health workers to reduce health disparities in Alabama's Black Belt: The pine apple heart disease and stroke project. Family \& Community Health, 29, 89-102. https://doi.org/10.1097/00003727-200604000-00005

Lebrun, L. A., Shi, L., Chowdhury, J., Sripipatana, A., Zhu, J., Sharma, R., ... Ngo-Metzger, Q. (2012). Primary care and public health activities in select US health centers: Documenting successes, barriers, and lessons learned. American Journal of Public Health, 102, S383-S391. https://doi.org/10.2105/AJPH.2012.300679

Lohr, A. M., Ingram, M., Nuñez, A. V., Reinschmidt, K. M., \& Carvajal, S. C. (2018). Community-clinical linkages with community health workers in the United States: A scoping review. Health Promotion Practice, 19, 349-360. https://doi.org/10.1177/1524839918754868

Miles, M. B., \& Huberman, A. M. (1994). Qualitative data analysis: An expanded sourcebook (2nd ed.). Thousand Oaks, CA: Sage.

Nath, J. B., Costigan, S., \& Hsia, R. Y. (2016). Changes in demographics of patients seen at federally qualified health centers, 2005-2014. JAMA Internal Medicine, 176, 712-714. https://doi.org/10.1001/jamaintern med.2016.0705

Nelson, S., Money, E. B., \& Petersen, R. (2016). Community health workers: An integral part of an integrated health care team. North Carolina Medical Journal, 77, 129-130. https://doi.org/10.18043/ncm.77.2.129

North Carolina Center for Health Statistics. (2017). Trends in key health indicators: North Carolina statewide and county. Retrieved July 25, 2017, from http://www.schs.state.nc.us/data/keyindicators/

O'Connor, A., \& Wellenius, G. (2012). Rural-urban disparities in the prevalence of diabetes and coronary heart disease. Public Health, 126, 813-820. https://doi.org/10.1016/j.puhe.2012.05.029

Patnode, C. D., Evans, C. V., Senger, C. A., Redmond, N., \& Lin, J. S. (2017). Behavioral counseling to promote a healthful diet and physical activity for cardiovascular disease prevention in adults without known cardiovascular disease risk factors: Updated evidence report and systematic review for the US Preventive Services Task Force. JAMA, 318, 175193. https://doi.org/10.1001/jama.2017.3303

Porterfield, D. S., Hinnant, L. W., Kane, H., Horne, J., McAleer, K., \& Roussel, A. (2012). Linkages between clinical practices and community organizations for prevention: A literature review and environmental scan. American Journal of Public Health, 102, S375-S382. https ://doi.org/10.2105/AJPH.2012.300692

Quigley, L., Matsuoka, K., Montgomery, K. L., Khanna, N., \& Nolan, T. (2014). Workforce development in Maryland to promote clinicalcommunity connections that advance payment and delivery reform. Journal of Health Care for the Poor and Underserved, 25(Suppl 1), 1929. https://doi.org/10.1353/hpu.2014.0062

Singh, G. K., \& Siahpush, M. (2014a). Widening rural-urban disparities in all-cause mortality and mortality from major causes of death in the USA, 1969-2009. Journal of Urban Health, 91, 272-292. https://doi. org/10.1007/s11524-013-9847-2

Singh, G. K., \& Siahpush, M. (2014b). Widening rural-urban disparities in life expectancy, U.S., 1969-2009. American Journal of Preventive Medicine, 46, e19-e29. https://doi.org/10.1016/j.amepre.2013.10.017

The Tri-Council for Nursing. (2017). The essential role of the registered nurse and integration of community health workers into community team-based care [Position statement]. Retrieved August 28, 2018, from https://tricouncilfornursing.org/position-statements

US Preventive Services Task Force. (2014). Healthful diet and physical activity for cardiovascular disease prevention in adults with cardiovascular risk factors: Behavioral counseling [Final recommendation statement]. Retrieved June 15, 2018, from https://www.uspreventiveservicestaskf orce.org/Page/Document/RecommendationStatementFinal/healthydiet-and-physical-activity-counseling-adults-with-high-risk-of-cvd

Williams, D. R., Priest, N., \& Anderson, N. B. (2016). Understanding associations among race, socioeconomic status, and health: Patterns and prospects. Health Psychology, 35, 407-411. https://doi.org/10.1037/hea0000242

How to cite this article: Allgood SD, Leeman J, Rosemond CA, Ammerman AS, Samuel-Hodge C, Cykert S. Reducing cardiovascular disease in a rural community. Public Health Nurs. 2019;36:676-682. https://doi.org/10.1111/phn.12649 